\title{
FLUCTUACIÓN POBLACIONAL DE PLAGAS INSECTILES EN EL CULTIVO DE VID VARIEDAD RED GLOBE, EN LA ZONA DE EL CARMEN - CHINCHA. PERÚ
}

\author{
POPULATION DYNAMICS OF INSECT PESTS ON "RED GLOBE” GRAPE VINE AT \\ "EL CARMEN" AREA - CHINCHA, PERU
}

${ }^{1}$ German Elias Joyo Coronado y ${ }^{2}$ Monica Narrea Cango

\begin{abstract}
Resumen
Se realizó una investigación para determinar la fluctuación poblacional de insectos plaga en el cultivo de vid (Vitis vinífera L.) variedad Red Globe, en El Carmen - Chincha, Perú. El estudio se desarrolló en un campo experimental de cinco hectáreas dividido en cinco sectores, en cada uno de las cuales fueron seleccionados aleatoriamente cinco plantas estratificándolas por cuadrantes, según la metodología recomendada por Sarmiento y Sánchez (2000).

Los resultados indican que el "enrollador de la hoja" Platynota sp. (Lep.: Tortricidae) se presentó desde el envero hasta la etapa de post-cosecha. El "esfíngido de la vid" Eumorpha vitis (L.) se registró en la etapa fenológica de postcosecha. La "filoxera de la vid" Daktulosphaera vitifoliae (Fitch), se observó sólo en la fase radicícola, alcanzando sus valores más altos de incidencia en la etapa posterior a la cosecha. Thrips tabaci Lindemann. y Frankliniella occidentalis (Pergande), se presentaron en las etapas fenológicas de floración y cuajado (fructificación).
\end{abstract}

Palabras claves: Daktulosphaera vitifoliae (Fitch), Eumorpha vitis (L.), Frankliniella occidentalis (Pergande), Platynota sp., Thrips tabaci Lindemann. y vid.

\begin{abstract}
Abstrac
A study was carried out to determine the population dynamics of insect pests in the cultivation of Vitis vinifera L., var. "Red Globe", located at "El Carmen" - Chincha, Peru. The study was conducted in an experimental field of five hectares divided in to five sectors, in each of which five plants were randomly selected, stratifying them into quadrants according to the method recommended by Sarmiento \& Sánchez (2000).Results indicate that the leaf roller Platynota sp. (Lep.: Tortricidae) presented itself from veraison up to post-harvest stage. The Vine Sphinx Moth Eumorpha vitis (L.) was registered in the phenological stage of post-harvest. The Grape Phylloxera, Daktulosphaera vitifoliae (Fitch), was detected only in its radicicle form, reaching its highest level of incidence in the phase subsequent to harvest. Thrips tabaci Lindemann and Frankliniella occidentalis (Pergande) presented them selves at the phenological stages of flowering and fruit set.
\end{abstract}

Key words: Daktulosphaera vitifoliae (Fitch), Eumorpha vitis (L.), Frankliniella occidentalis (Pergande), Platynota sp., Thrips tabaci Lindemann and grape vine.

\section{Introducción}

Se puede encontrar en el cultivo de la vid, una gran diversidad de insectos como; Platynota sp. , Eumorpha vitis L., Daktulosphaera vitifoliae (Fitch.), Thrips tabaci lindemann, Frankliniella occidentalis (Pergande), Ceratitis capitata (Wied.), Planococcus citri Risso, Hemiberlesia lataniae Signoret, Anomala undulata Melsheimer, Parepitragus pulverulentus Gebien y otros; los cuales se encuentran distribuidos en todas las zonas vitícolas, causando pérdidas económicas, debido a los daños directos e indirectos en la producción.

Según Fennah (1947), Platynota sp (Lepidoptera: Tortricidae), es conocido como "enrollador de la hoja" comúnmente relacionado a cultivos de leguminosas. En nuestro país para el cultivo de vid es citado por Alata (1973); quien además registra esta especie para otros cultivos como lino, quina, leguminosas, fresa, mango, palto y trigo. El mismo autor cita a Platynota rostrana (Walk.) en el cultivo de algodonero. En algunas regiones de Brasil, las larvas de esta especie han sido registradas, en cítricos en el periodo inmediato a la floración, infestando frutos maduros e inmaduros (Nava et al., 2006).

Eumorpha vitis L. (Lepidoptera: Sphingidae), es de escasa importancia económica, los adultos son de actividad nocturna, ovipositan aisladamente en las hojas tiernas, las larvas son voraces consumiendo una gran cantidad de área

\footnotetext{
${ }^{1}$ Universidad Nacional Agraria La Molina, Liam, Perú. Email: gjoyo@lamolina.edu.pe

${ }^{2}$ Universidad Nacional Agraria La Molina, Liam, Perú. Email: mnarrea@lamolina.edu.pe
} 
foliar durante todo su desarrollo. Las altas infestaciones generalmente se han observado luego de la cosecha, esto es en la brotación que se genera luego de la fertilización y riego en la etapa de postcosecha, especialmente en vides de exportación (Sánchez y Vergara, 2003).

Según Granett et al (2001), la "filoxera" Daktulosphaera vitifoliae (Fitch.), se alimenta de los brotes y las raíces, siendo en estas últimas donde ocasiona las mayores daños, generando nudosidades resultantes de la hipertrofias de las raíces fibrosas, que reducen la capacidad de la planta para absorber los nutrientes. Como daño secundario, las heridas de las hipertrofias sirven como puerta de entrada a los hongos del suelo que causan la pudrición de la raíz, que puede acarrear la muerte de la planta. De manera similar Sánchez y Vergara (2003), indican que presenta dos fases definidas de infestación en la vid. La primera considerada fase radicícola, que daña las raices, y la segunda fase gallícola, que daña las hojas.

Según Sánchez y Vergara (2003), las infestaciones de Thrips tabaci Lindemann y Frankliniella occidentalis (Pergande) en el cultivo de vid, se pueden dar desde el inicio del brotamiento. Así mismo señalan que, los daños en los brotes se traducen en raspaduras y succión de la savia, apareciendo los brotes con el aspecto de quemado. En los frutos recién cuajados se observan rajaduras, los que se manifiestan en manchas relativamente grandes en frutos desarrollados. En caso de daños severos al cuajado, los frutos conforme se desarrollan, presentan rajaduras que afectan la calidad de los granos y pueden propiciar el desarrollo del hongo Botrytis cinerea. Alvarado et al (1990), menciona que Frankliniella occidentalis (Pergande) puede ocasionar deformaciones en los brotes, caída de flores y un envejecimiento precoz.

Los daños ocasionados por Ceratitis capitata (wied.) pueden ser de tipo directo, por la oviposición de las hembras al depositar sus huevecillos en los frutos, alimentación de las larvas de la pulpa del fruto y caída de los frutos infestados, e indirectos; por la pérdida del valor comercial de los frutos infestados, gastos en la aplicación de cebos tóxicos, disminución del rendimiento y la producción, y restricción en el comercio internacional por constituir plagas cuarentenarias (SENASA, 1997).

Considerando la escasa información sobre la ocurrencia de plagas insectiles en la vid, se estimó conveniente subsanar esta omisión con la presente investigación, cuyo objetivo fue determinar la fluctuación poblacional de las plagas en el cultivo de vid, variedad Red Globe, zona del Carmen - Chincha.

\section{Materiales y métodos}

Este trabajo fue realizado en un campo agrícola del cultivo de vid (Vitis vinífera L.), variedad Red Globe de 5 hectáreas, perteneciente a la empresa Copacabana ubicado en el distrito El Carmen, Provincia Chincha, Departamento de Ica, con coordenadas geográficas $13^{\circ}$ $25^{\prime} 29^{\prime}$ " latitud sur y $76^{\circ} 08^{\prime} 01$ ' Longitud oeste; altitud
$97 \mathrm{msnm}$. Se realizaron un total de 43 evaluaciones (una por semana) abarcando las diferentes etapas fenológicas del cultivo.

Las evaluaciones se realizaron siguiendo la metodología citada por Sarmiento y Sánchez (2000), para esto el campo se dividió en cinco sectores, en cada sector se evaluó 5 plantas representativas al azar, las cuales se estratificaron por cuadrantes.

En cada planta seleccionada se observó; cuatro brotes, cuatro hojas, cuatro inflorescencias y cuatro racimos (una por cada cuadrante) examinando en total 100 ramas, 100 brotes, 100 hojas, 100 inflorescencias y 100 racimos.

Durante el cultivo de vid se realizaron siete tratamientos químicos en las fechas $\mathrm{y}$ con principios activos siguientes: 06 y 23 de febrero, carbofuran (6 y 4 L/ha respectivamente); 23 de marzo, 14 de abril, 12 de agosto y 08 de setiembre, cyfluthrina en mezcla con metamidofos (1.5 L/ha) y el 08 de octubre, carbosulfan $(0.8 \mathrm{~L} / \mathrm{ha})$.

Los registros de temperatura y humedad relativa fueron obtenidos de la estación meteorológica propia de la empresa.

El sistema de evaluación utilizada para cada una de las especies plagas fue la siguiente:

\subsection{Evaluacion de Platynota sp.}

En cada cuadrante se revisó una rama o brazo, del cual se evaluó una hoja y un racimo, a razón de 20 hojas y 20 racimos por sector, registrándose en total 100 hojas y 100 racimos. Semanalmente se registro el número total de larvas en 25 plantas y el porcentaje de plantas infestadas.

\subsection{Evaluacion de Eumorpha vitis L.}

En cada cuadrante se tomó una rama o brazo, del cual se evaluó una hoja y un brote, a razón de 20 brotes y 20 hojas por sector, registrándose en total 100 brotes y 100 hojas. Semanalmente se registró el número de larvas totales en 25 plantas y el número de hojas dañadas.

\subsection{Evaluación de Daktulosphaira vitifoliae (Fitch).}

En cada cuadrante se observó una rama o brazo, del cual se evaluó una hoja y un brote, a razón de 20 brotes y 20 hojas por sector, registrándose en total 100 brotes y 100 hojas. Se anotó si la hoja y el brote estaban sanos o infestados.

Para determinar la presencia de D. vitifoliae (Fitch), en el sistema radicular, se efectuaron calicatas en cuatro ocasiones después de la cosecha. Así la primera observación se realizó el 16 de marzo y las tres últimas después de la poda: 10 de agosto, 14 y 21 de setiembre. Previamente se seleccionaron 10 plantas, a razón de 2 plantas por sector, y como muestra se tomó 5 centímetros de raíz más raicillas, donde se observó y registró el número de adultos y ninfas de esta especie. 


\subsection{Evaluación de Thrips tabaci Lindemann y Frankliniella occidentalis (Pergande)}

En cada cuadrante se observó una rama o brazo, del cual se evaluó un brote y una inflorescencia, a razón de 20 brotes y 20 inflorescencias por sector, registrándose en total 100 brotes y 100 inflorescencias. Los brotes e inflorescencias se sacudieron sobre una superficie de color blanco, anotando el número total de trips en 25 plantas y el porcentaje de plantas infestadas.

\subsection{Evaluación de Ceratitis capitata (Wied.)}

Para la evaluación de la mosca adulta se utilizó 5 trampas con atrayentes de alimentación, una trampa por cada zona. Las trampas consistieron de recipientes de polipropileno de 2.5 litros de capacidad, con agujeros en los márgenes superiores, a los cuales se les adicionó una solución a base de 20 litros de agua más 1.5 kilos de fosfato diamónico. En cada evaluación se registró el número de moscas capturadas por trampa.

\section{Resultados y discusión}

\subsection{Fluctuación poblacional de Platynota sp.}

La fluctuación poblacional de Platynota sp. se presenta en la fig. 1. El mayor registro de este fitófago correspondió al día 07 de diciembre, fecha en la cual se observó 186 larvas en 25 plantas de vid y $100 \%$ de plantas infestadas. Las evaluaciones de los días 26 de enero y
09 de febrero también registraron altas infestaciones, siendo estas 156 y 169 larvas respectivamente y 100 $\%$ de plantas infestadas. Como se observa, la época de mayor infestación correspondió a los meses de verano con temperaturas medias que fluctuaron entre 22.6 y 30.5 ${ }^{\circ} \mathrm{C}$ y humedad relativa media entre 70.2 y $79.7 \%$, este periodo coincidió con las etapas fenológicas de finales de pinta, maduración y postcosecha.

En la fig. 01 también se observa que después de la evaluación del 07 de diciembre ocurrió una disminución en el número de larvas, llegando a registrarse en la evaluación del 05 de enero, 27 larvas en 25 plantas y 84 $\%$ de plantas infestadas. Posterior a esta semana, debido al aumento de temperatura, nuevamente se incrementó el número de larvas infestantes.

En las observaciones del 26 de enero, 02 y 09 de febrero se observaron 3 registros altos de población larval con 156, 121 y 169 larvas en 25 plantas respectivamente y $100 \%$ de plantas infestadas. Semanas después, debido a la aplicación de insecticidas, la población del enrollador disminuyó considerablemente registrando el 20 de abril, sólo 1 larva en 25 plantas evaluadas y $4 \%$ de plantas infestadas. Posteriormente con las aplicaciones de insecticidas de los días 12 de agosto, 08 de setiembre y 08 de octubre; no se registró una nueva infestación.

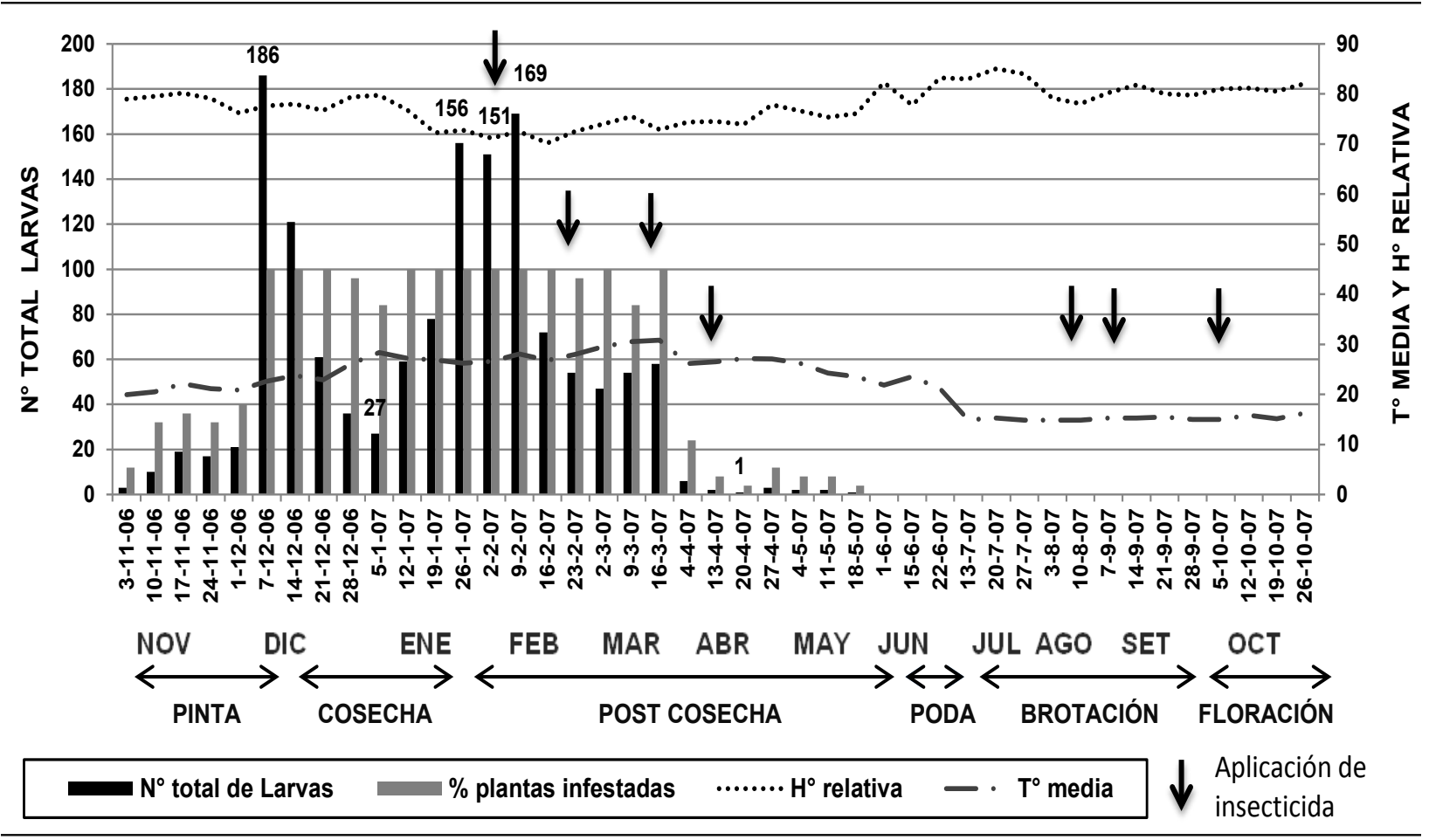

Figura 1. Fluctuación poblacional de larvas de Platynota sp. (Lepidoptera: Tortricidae) en vid, Variedad Red Globe. Chincha-Perú. 


\subsection{Fluctuación poblacional de Eumorpha vitis $\mathbf{L}$.}

Los resultados se presentan en la fig. 2, como se puede apreciar, el primer registro de larvas infestantes ocurrió el 05 de enero, fecha en la cual se observó 01 larva en 25 plantas de vid y 20 hojas dañadas. La presencia de larvas infestantes, que ocurre a partir de la etapa fenológica de postcosecha, se debió a que durante y después de la cosecha no se aplicó insecticidas. La presencia de los daños realizados por esta especie se observó entre los meses de diciembre hasta fines de marzo, con temperaturas medias que fluctuaron entre 22.6 y $30.5{ }^{\circ} \mathrm{C}$ y humedad relativa media entre 70.2 y $79.7 \%$, este periodo coincidió con las etapas fenológicas de finales de pinta, maduración y postcosecha.
Los mayores registros de esta especie correspondieron al mes de marzo en la etapa fenológica de postcosecha, coincidiendo con lo observado por Sánchez (2003), quien considera a este lepidóptero como de escasa importancia económica. En el periodo mencionado se observó el 02 de marzo 12 larvas en 25 plantas de vid y 41 hojas dañadas; el 09 de marzo, 13 larvas y 37 hojas dañadas; y el 16 de marzo, 11 larvas y 31 hojas dañadas.

Después del mes de marzo la densidad poblacional de este fitófago sufrió una caída abrupta, registrándose el 04 de abril 01 larva en 25 plantas de vid. El declive de la curva poblacional y la ausencia de esta especie en las etapas fenológicas posteriores ocurrieron debido a las aplicaciones de insecticidas realizadas el 23 de marzo y 14 de abril.

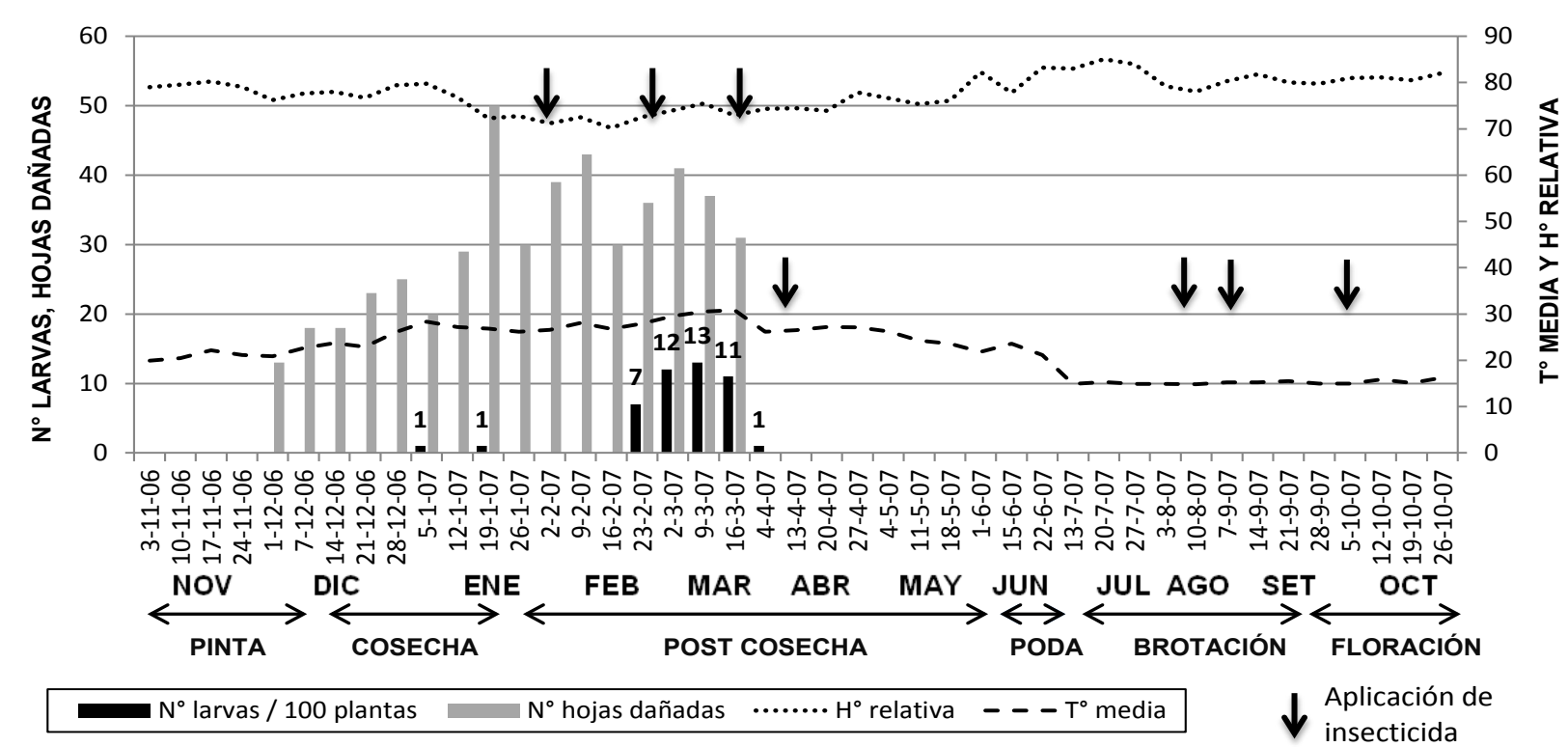

Figura 2. Fluctuación poblacional de larvas y número de hojas dañadas por Eumorpha vitis L., en vid, variedad Red Globe. Chincha - Perú.

\subsection{Fluctuación poblacional Daktulosphaera vitifoliae (Fitch.)}

Durante las evaluaciones realizadas en las plantas de vid no se registró la presencia de la filoxera en hojas, es decir en su fase gallícola.

El cuadro 1 y la fig. 3 muestran los resultados de las evaluaciones realizadas en la parte radicular, en las cuales se observó que en el mes de marzo, periodo correspondiente a la etapa de postcosecha, se presentó el mayor registro de "filoxera" en su fase radícola; registrando 115 individuos en 10 plantas de vid y 10 plantas infestadas.

Las evaluaciones realizadas posterior a la poda registraron una baja en la población de más de 75 por ciento, observando el 10 de agosto 25 individuos en 10 plantas de vid y 3 plantas infestadas, el 14 de setiembre se registró 13 individuos y 3 plantas infestadas; y finalmente el 21 de setiembre se observó 16 individuos y 4 plantas infestadas.
Aun cuando no se tienen registros para las etapas fenológicas de fructificación y maduración, los obtenidos en el mes de marzo, correspondientes a la post cosecha (fig. 3) demostraron que este insecto fue incrementando su población después de la etapa fenológica de brotación.

Cuadro 1. Número de adultos + ninfas y de plantas infestadas por Daktulosphaera vitifoliae (Fitch.) en raíces de vid, variedad Red Globe. Chincha - Perú.

\begin{tabular}{lcc}
\hline Fecha & $\mathrm{N}^{\circ}$ Total ninfas y adultos & $\begin{array}{c}\mathrm{N}^{\circ} \text { Plantas } \\
\text { infestadas }\end{array}$ \\
\hline 16 -mar & 115 & 10 \\
10 -ag & 25 & 3 \\
14 -sep & 13 & 3 \\
21 -sep & 16 & 4 \\
Total & 169 & 20 \\
\hline
\end{tabular}




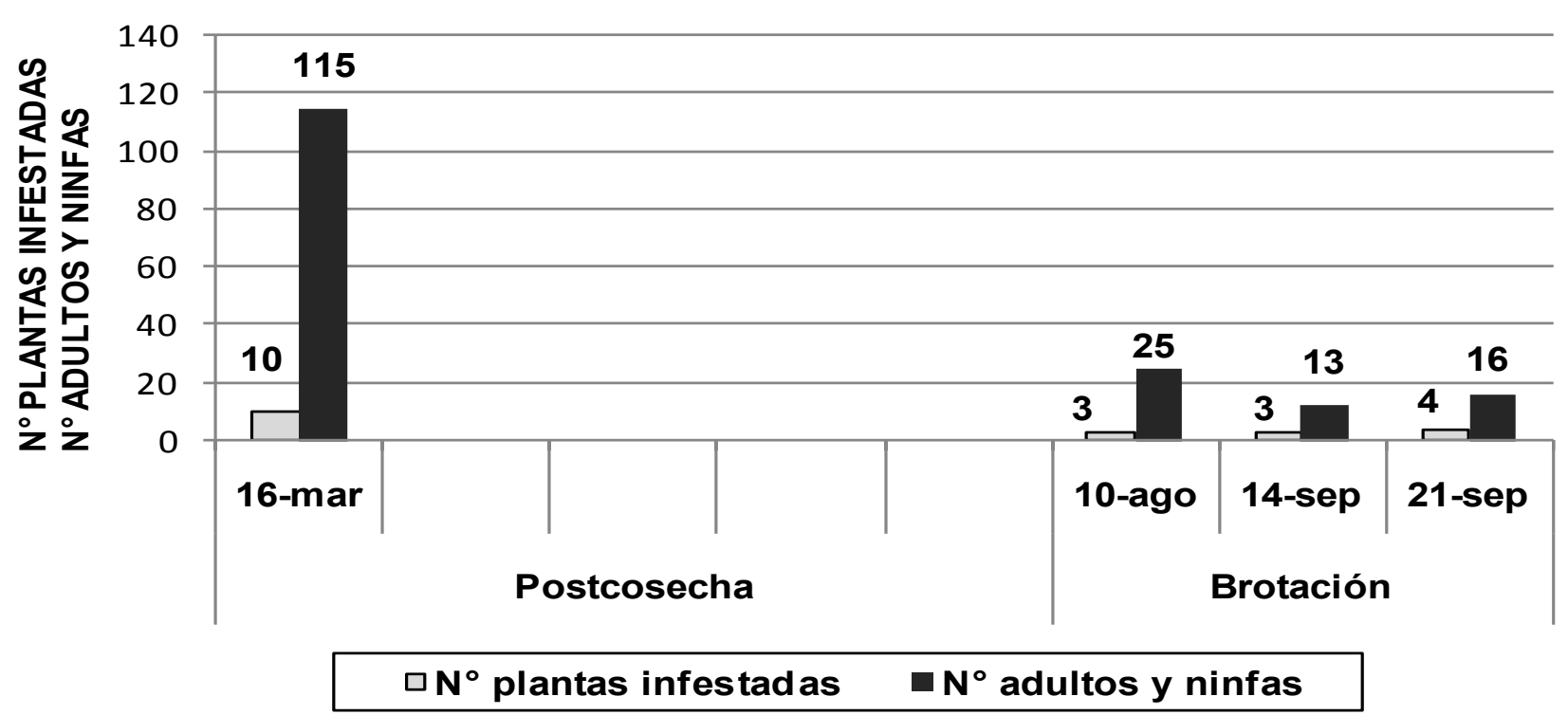

Figura 3. Número de adultos + ninfas de Daktulosphaera vitifoliae (Fitch.) en raíces de vid, variedad Red Globe. Chincha - Perú.

Como lo demuestran los resultados, las aplicaciones de insecticidas dirigidas a la parte radicular (carbofuran), además de los utilizados en la parte aérea para el control de trips y Platynota sp., evitó la presencia de este insecto fitófago en el área foliar (fase gallícola), y mantuvo baja la población en el sistema radicular en las etapas fenológicas de brotación y floración, etapas en las cuales es muy importante mantener bajas las poblaciones de los insectos fitófagos para obtener una adecuada producción.

\subsection{Fluctuación poblacional de trips}

Las especies colectadas en el campo experimental fueron Thrips tabaci lindemann y Frankliniella occidentalis (Pergande) (identificadas en el museo de entomología "Klaus Raven Büller").

La fig. 4 muestra el número de ninfas y adultos de ambas especies de trips y el porcentaje de infestación registrados durante el periodo de evaluación. Según estos resultados son pocas las evaluaciones de campo en las que se registra la presencia de esta plaga.

Los mayores registros se observaron en la etapa fenológica de pinta, con temperaturas medias que fluctuaron entre 19.9 y $22.1{ }^{\circ} \mathrm{C}$ y humedad relativa promedio entre 76.3 y $80.2 \%$. Así se observó el 03 de noviembre 88 individuos en 25 plantas de vid y $52 \%$ de plantas infestadas. A partir del mes de diciembre, a finales de la etapa fenológica de pinta e inicios de cosecha, no se registró la presencia de este fitófago en planta hasta el mes de mayo, en la etapa de postcosecha, donde únicamente se registró en la segunda semana 2 individuos en 25 plantas. Posteriormente se volvió a registrar trips en la segunda semana del mes de octubre, en la etapa fenológica de floración, observando 5 individuos en 25 plantas y $20 \%$ de plantas infestadas; finalmente en la cuarta semana del mes de octubre se observó 15 individuos en 25 plantas y $36 \%$ de plantas infestadas.

La aparición de trips en las plantas de vid, a partir del mes de octubre, ocurrió como respuesta a la aparición de la floración, tal como lo demuestra el trabajo realizado por Mamani (2009) en el cultivo de alcachofa, quien registró la mayor población de Thrips tabaci Lindemann, 775 y 1299 individuos en 25 plantas, en los meses de junio y agosto respectivamente; relacionado a la etapa fenológica de formación del capítulo floral.

En los registros de trips en la etapa fenológica de floración se observó la presencia de muy pocos adultos y ninfas de trips, debido a las dos aplicaciones de insecticidas realizadas en la etapa de post cosecha en los meses de marzo y abril para el control de Platynota sp.; y las tres aplicaciones de insecticidas realizadas después de la poda en los meses de agosto, septiembre y octubre. Estas aplicaciones evitaron la presencia de adultos y ninfas de trips en las etapas de post cosecha, poda y brotamiento (periodo en que la población de este esta plaga fue muy baja), y redujo su presencia en la etapa de floración, etapa en la cual según Sanchez y Vergara (2003) se presentan las mayores infestaciones.

Otro factor que estuvo relacionado a la ausencia y baja población de trips en la floración; fue la menor temperatura registrada en los meses posteriores a la poda, registrándose temperaturas medias que fluctuaron entre $14.84{ }^{\circ} \mathrm{C}$ y $16.82{ }^{\circ} \mathrm{C}$, a diferencia de las temperaturas medias para los mismos meses en el año anterior, las cuales fluctuaron entre $16.84{ }^{\circ} \mathrm{C}$ y $19.05{ }^{\circ} \mathrm{C}$, siendo estas mayores en más de $2{ }^{\circ} \mathrm{C}$. Este hecho retrasó la brotación y la floración dos a tres semanas más de lo normal; influyendo también en la escasa población de adultos y ninfas de trips. 


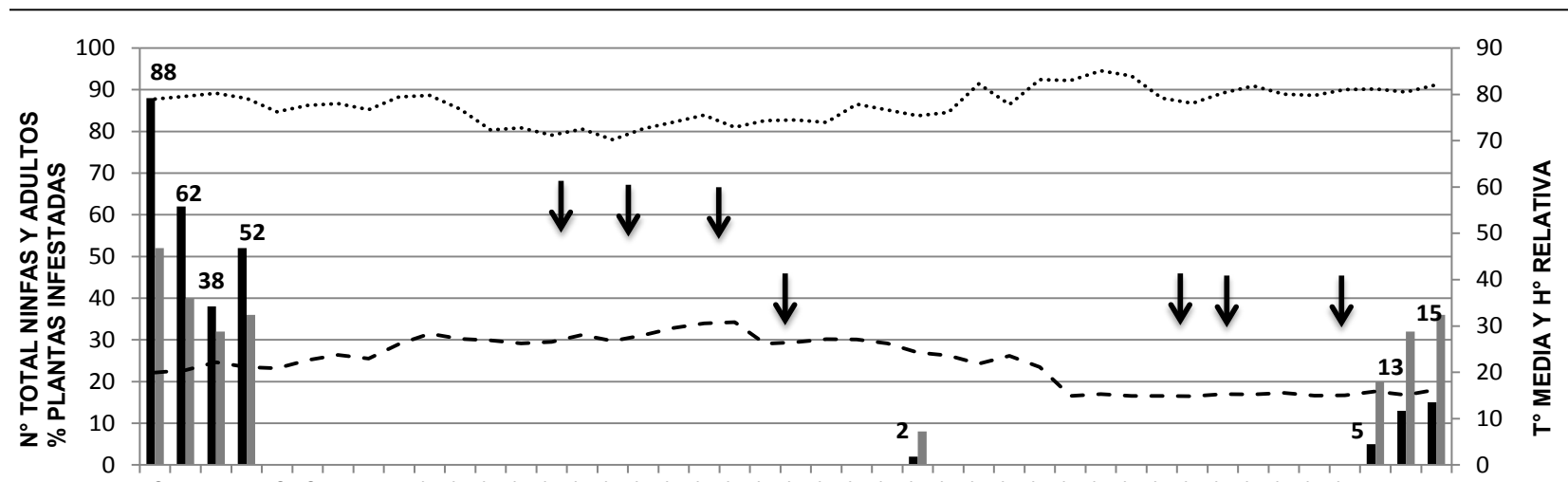

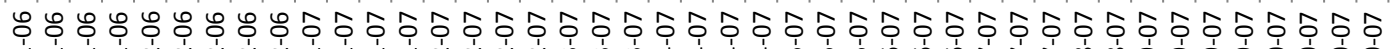

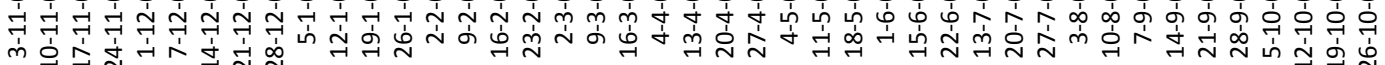

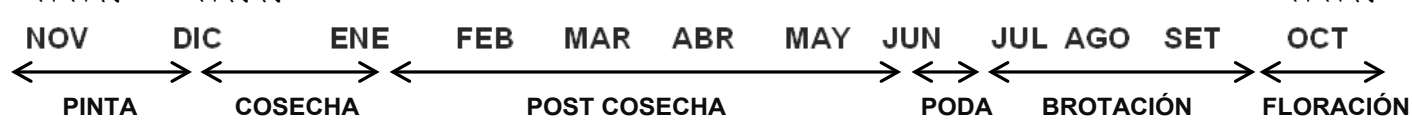

$\mathrm{N}^{\circ}$ Total ninfas y adultos $\quad \%$ Plantas infestadas $\quad \cdots \cdots . . . . . \mathrm{H}^{\circ}$ relativa $\quad$ - $--\mathrm{T}^{\circ}$ media

Figura 4. Fluctuación poblacional de ninfas + adultos de trips y porcentaje de plantas infestadas de vid, variedad Red Globe. Chincha - Perú.

\subsection{Fluctuación poblacional de Ceratitis capitata (Wied.)}

La fig. 5 muestra las evaluaciones realizadas para registrar la presencia de Ceratitis capitata (Wied.), el único registro correspondió a la evaluación realizada el 23 de feb

rero, fecha en la cual sólo se encontró un adulto. A pesar de que la presencia de este individuo ocurrió en la etapa fenológica de postcosecha de vid, tuvo relación con la época de cosecha de mandarina (cultivo ubicado a más de medio kilómetro del campo de evaluación).
La ausencia de moscas de la fruta se debió, además del buen manejo que ejecutó esta empresa en el cultivo de mandarina, al tipo de atrayente utilizado en las trampas y a las aplicaciones de insecticidas realizadas en el campo de vid. Otro factor fue la distancia entre los campos de cítricos y el lote de vid evaluado, y finalmente la presencia de cortinas de vientos de arpillera y árboles de álamo entre los campos de cítricos y los de vid.

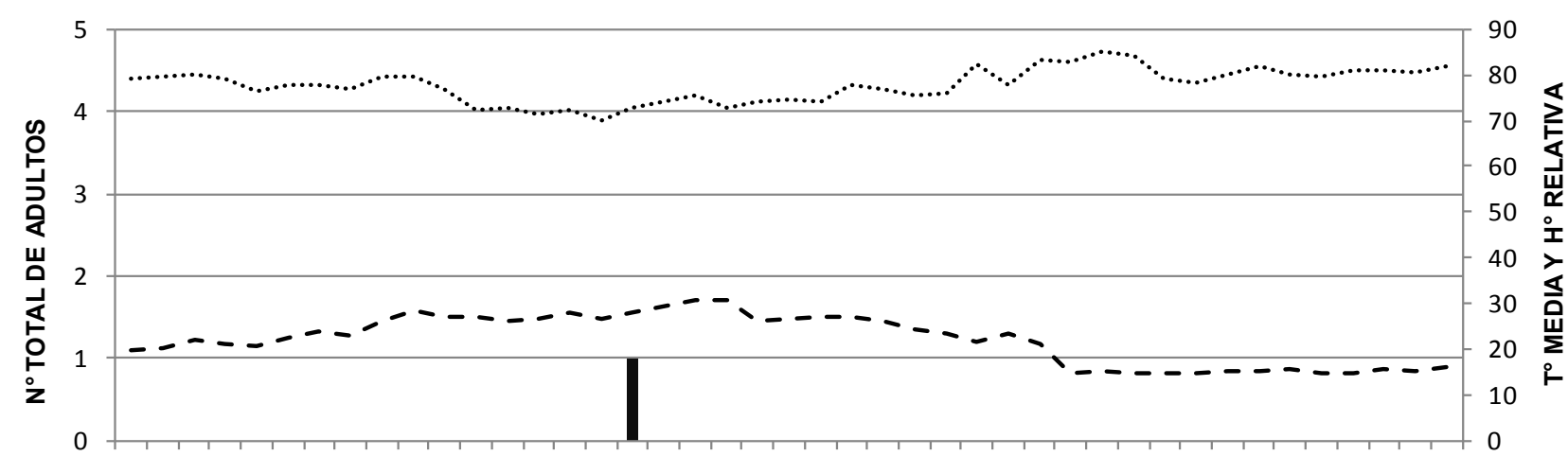

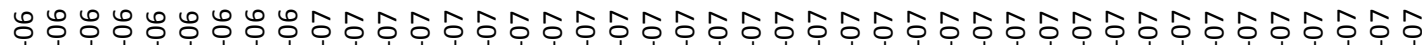

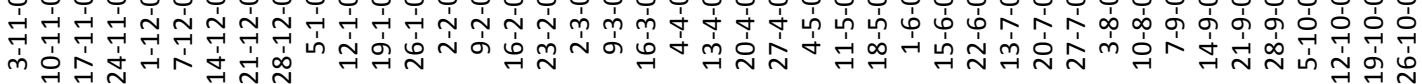

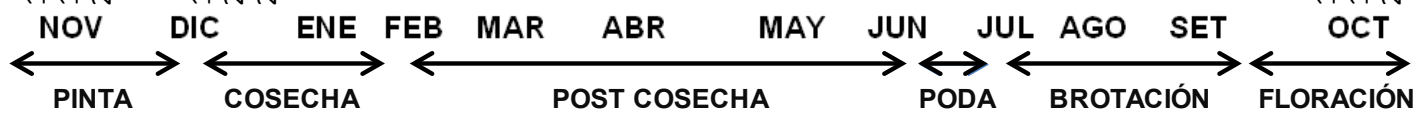

$\mathrm{N}^{\circ}$ total de adultos $\quad \cdots \cdots \cdots \cdot \mathrm{H}^{\circ}$ relativa $\quad-\cdots-\mathrm{T}^{\circ}$ media

Figura 5. Fluctuación poblacional de adultos de Ceratitis capitata (Wied.) en vid, variedad Red Globe. Chincha Perú. 


\section{Conclusiones}

Bajo las condiciones en la cuales se realizaron las evaluaciones se obtienen las siguientes conclusiones:

Platynota sp. se presenta en el cultivo de vid en en el fundo Copacabana en todas las etapas fenológicas dañando brotes, hojas y bayas de los racimos de vid.

Las larvas y daños de Eumorpha vitis L., se presentan entre los meses de diciembre y abril, en el periodo de postcosecha.

La "filoxera", Daktulosphaera vitifoliae (Fitch.), en su fase gallícola, no se presenta infestando hojas, a diferencia de su fase radicícola, que presenta su mayor infestación en los meses posteriores a la cosecha.

Las especies Thrips tabaci Lindemann. y Frankliniella occidentalis (Pergande), se presentan en las etapas fenológicas de floración y cuajado (fructificación).

\section{Literatura citada}

Alata C, J. 1973. Lista de insectos y otros animales dañinos a la agricultura en el Perú. Ministerio de Agricultura. Manual 38. Dirección General de Investigación Agraria, Centro de Investigación Agraria $N^{\circ}$ 1. Estación Experimental La Molina, Departamento de Entomología. Lima Perú. 175 p.

Alvarado, M.; Serrano, A.; De La Rosa, A. y Berlanga, M. 1990. Frankliniella occidentalis en melocotón y nectarina de Andalucía occidental. Phytoma España, 21: 51-55.

Bautista, D. 1995. Factores favorables para el cultivo tropical de la vid. Trabajo de ascenso. Decanato de Agronomía. Universidad Centroccidental Lisandro Alvarado. Barquisimeto, Venezuela. 156 p.

Fennah, R. G. 1947. The insect pests of food crops in the lesser Antilles. Dep. Agric. Antigua BWI. 207 p.

Granett, J.; Walker, M. A. y Kocsis, L. 2001. Biology and management of grape

phylloxera. Annual Review of Entomology, 46: 387-412.

Mamani, D. 2009. Fluctuación poblacional de los principales insectos fitófagos y sus enemigos naturales en el cultivo de alcachofa en el valle de Ica. Tesis para optar el grado de Magíster Scientiae. Universidad Nacional Agraria La Molina. Lima-Perú. 124 p.

Nava, D. E.; Fortes, D.G. D.; Olivera, F. T; Viera, T.M. Ibelli, F. J. V. C. Guedes and Parra, J. R. 2006. Platynota rostrana (Walker) (Tortricidae) and Phidotricha erigens Raganot (Pyralidae): artificial diet effects on biological cycle. Brazilian Journal of Biology, 66(4): 1037-1043.

Sánchez, G. y Vergara, C. 2003. Plagas de los frutales. Universidad Nacional Agraria La Molina. Lima-Perú. 193 p.

Sarmiento, J. y Sánchez, G. 2000. Evaluación de insectos. Universidad Nacional Agraria La Molina. Lima-Perú. 160 p.
SENASA (Servicio Nacional de Sanidad Agraria, PE). 1997. Control integrado de las moscas de la fruta. Programa Nacional de Moscas de la fruta. SENASA, MINAG. 54 p. 